\title{
Study of twinned dendrite growth stability
}

\author{
M.A. Salgado-Ordorica, ${ }^{*}$ J. Valloton and M. Rappaz \\ Laboratoire de Simulation des Matériaux, Ecole Polytechnique Fédérale de Lausanne, CH-1015 Lausanne, Switzerland
}

Received 17 March 2009; revised 9 April 2009; accepted 9 April 2009

Available online 14 April 2009

\begin{abstract}
Under certain thermal conditions $\left(G \approx 1 \times 10^{4} \mathrm{~K} \mathrm{~m}^{-1}, v_{\mathrm{s}} \approx 1 \times 10^{-3} \mathrm{~m} \mathrm{~s}^{-1}\right),\left\langle\begin{array}{llll}1 & 1 & 0\rangle\end{array}\right.$ twinned dendrites appear in aluminum alloys and can overgrow regular columnar dendrites, provided that some convection is also present in the melt. In order to check the stability of such morphologies, directionally solidified twinned samples of Al-Zn were partially remelted in a Bridgman furnace and then resolidified under controlled conditions, with minimal convection. It was found that, although twin planes remain stable during partial remelting, non-twinned dendrites regrow during solidification. They have a crystallographic orientation given by those of the twinned and untwinned "seed" regions, and grow along preferred directions that tend to be those of normal specimens. (c) 2009 Acta Materialia Inc. Published by Elsevier Ltd. All rights reserved.
\end{abstract}

Keywords: Bridgman solidification; Dendrite growth; Twin stability; Aluminum alloys

Feathery grains were found for the first time as a defect in semi-continuous casting of aluminum alloys almost 60 years ago [1]. Using electron backscattered diffraction (EBSD), Henry et al. [2-4] clearly showed that these fan-like structures are made of a lamellar sequence of twinned and untwinned regions with dendrites split in the center of their trunk by a coherent $\left\{\begin{array}{llll}1 & 1 & 1\end{array}\right\}$ twin plane. Twinned dendrite trunks always grow along $\left\langle\begin{array}{llll}1 & 1 & 0\end{array}\right)$ directions and have a highly complex branch morphology of $\langle 110\rangle$, and also sometimes $\langle 100\rangle$, secondary arms [5]. ${ }^{1}$ Experience has shown that such morphologies form when the following twinning conditions are met: (i) a fairly high thermal gradient $\left(G \approx 1 \times 10^{4} \mathrm{~K} \mathrm{~m}^{-1}\right)$; (ii) a fairly large solidification rate $\left(v_{\mathrm{s}} \approx 1 \times 10^{-3} \mathrm{~m} \mathrm{~s}^{-1}\right)$; and (iii) some convection present in the melt $[5,7]$. Such conditions seem to give a kinetic advantage to twinned grains over ordinary columnar grains, i.e. under the same solidification conditions, the undercooling of the twinned dendrite tips, $T_{t w}$, are lower than that of regular columnar dendrites, $T_{r c}$ $[5,8-10]$. However, the morphology of the twinned dendrite tip is itself still an open question. A grooved tip [8], an edgy tip [9] and a deep-grooved tip (called in this case "doublon") [10] have all been proposed by different authors. Furthermore, the influence of convection has

\footnotetext{
*Corresponding author. Tel.: +41 21 6932979; e-mail addresses: mario.salgado@epfl.ch; michel.rappaz@epfl.ch

${ }^{1}$ In the field of rapid solidification processes (undercoolings more than one order of magnitude higher than those considered here), twinned dendrites growing along $\left\langle\begin{array}{llll}1 & 1 & 1\end{array}\right\rangle$ directions have been reported [6].
}

been clearly identified [7], but the detailed mechanism by which this operates is still unclear. Does convection only act on the nucleation stage via an enhanced formation of stacking faults? Is it also necessary during growth? In order to answer such questions, specimens produced under twinning conditions were partially remelted and then resolidified under well-controlled Bridgman conditions, for which convection in the melt is minimal.

With this goal in mind, a binary aluminum alloy of composition Al-23 wt. $\% \mathrm{Zn}$ was selected for three reasons: (i) it has been shown recently that twins can form easily in this system with up to about $40 \mathrm{wt} . \% \mathrm{Zn}$ [5]; (ii) zinc being heavier than aluminum and the partition coefficient $k_{0}$ being smaller than one, solidification in a vertical thermal gradient minimizes solutal (and thermal) natural convection; and (iii) zinc can influence the growth directions of "regular" aluminum dendrites as a function of the composition [11]. By "regular" we mean, in the following of the text, dendrites which are untwinned, regardless of their growth direction. Therefore, the regrowth of dendrites from a twinned seed can also supply interesting information.

The specimens were prepared from pure elements (99.995 wt.\%), then poured and directionally solidified (DS) in a cylindrical mold of $55 \mathrm{~mm}$ diameter and $150 \mathrm{~mm}$ height. Details of this experimental setup can be found in Refs. [5,11]. After solidification, cylindrical specimens of $5 \mathrm{~mm}$ diameter and nearly $150 \mathrm{~mm}$ length were machined out of the DS ingots in such a way that the axis was nearly parallel to the twin plane of a single feathery grain. These small cylinders were then introduced into an alumina crucible $(5 / 9 \mathrm{~mm}$ inner/outer diameter), which 
was placed in the water-cooled region of a two-zone Bridgman furnace (described in Ref. [11]). After raising the temperature of the furnace to $500{ }^{\circ} \mathrm{C}$, the sample was pulled up into the hot region at time $t_{0}$. The temperature of the furnace was then increased up to $760{ }^{\circ} \mathrm{C}$ in order to ensure partial remelting of the sample. The remelting operation was controlled with the help of thermocouples placed within the specimen and within the mold. At time $t_{\mathrm{p}}$, the specimen was either directly quenched into a water container located below the Bridgman furnace, in order to simply visualize the solid-liquid interface after remelting, or resolidified at a controlled velocity $v_{\mathrm{p}}$ by pulling the specimen downward a certain distance before quenching at time $t_{\mathrm{q}}$ (typically $v_{\mathrm{p}}=6.6 \times 10^{-5}$ and $3.3 \times 10^{-4} \mathrm{~m} \mathrm{~s}^{-1}$. The measured temperatures were also used as an input to inverse heat transfer simulations performed with the software CALCOSOFT $^{\circledR}$. This allowed the deduction of the temperature profile in both the specimen and the crucible between $t_{0}$ and $t_{\mathrm{p}}$, where $\left(t_{\mathrm{p}}-t_{0}\right)$ is about $10 \mathrm{~min}$. From these calculations, it was deduced that: (i) the longitudinal thermal gradient $G$ inside the specimen is about $2.5 \times 10^{3} \mathrm{~K} \mathrm{~m}^{-1}$; and (ii) the radial thermal gradient is weak, typically $10 \mathrm{~K} \mathrm{~m}^{-1}$, thus minimizing natural convection for this alloy. Therefore, the present Bridgman experiment deviates from the standard twinning conditions of DS in the sense that both convection and thermal conditions $\left(G\right.$ and $\left.v_{\mathrm{p}}\right)$ are reduced.

Figure 1 shows two transverse micrographs (i.e. perpendicular to the thermal gradient) of a DS Al-23 wt.\% Zn ingot. It exhibits regions where twinned (a) and regular columnar (b) grains have grown. In (a), rows of twinned dendrite trunks can be clearly identified: they correspond to traces of $\left\{\begin{array}{lll}1 & 1 & 1\end{array}\right\}$ planes in this transverse section [5]. The spacing between two successive rows, $\lambda_{1 \perp}$, is $\sim 200 \mu \mathrm{m}$, whereas the trunk spacing within a row, $\lambda_{1 \|}$, is only $\sim 100 \mu \mathrm{m}$. This shows that the solute diffusion field around a twinned dendrite tip and its secondary arms exhibits an asymmetry. In the regular columnar grain (Fig. 1b), the dendrites appear in this transverse section as crosses typical of $\left\langle\begin{array}{lll}1 & 0 & 0\end{array}\right\rangle$ growth with a fairly uniform spacing $\lambda_{1} \approx 100 \mu \mathrm{m}$. It is tempting to use the non-uniform dendrite trunk spacing of twinned grains to draw some conclusions regarding their growth advantage over regular dendritic ones. Unfortunately, the primary spacing is influenced not only by the dendrite tip shape itself, but also by the morphology/configuration of secondary arms, which are markedly different in the two grains of Figure 1. In the case of the twinned grain shown in Figure $1 \mathrm{a}$, secondary arms are growing along $\left\langle\begin{array}{llll}1 & 1 & 0\end{array}\right\rangle$ rather than $\left\langle\begin{array}{llll}1 & 0 & 0\end{array}\right\rangle$ directions. Therefore, they are not symmetrically

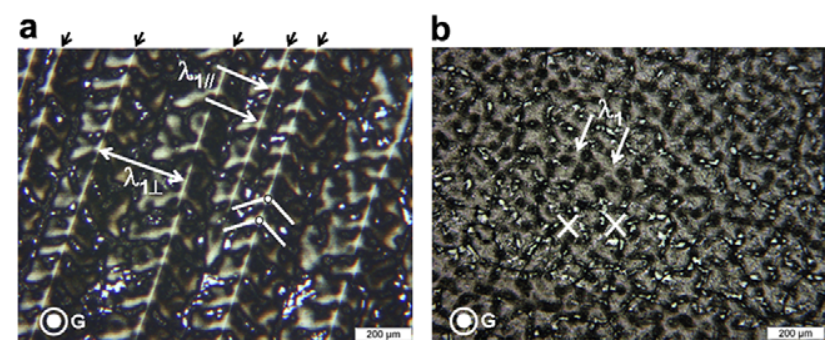

Figure 1. Transverse sections of twinned (a) and regular columnar (b) grains in a DS Al-23 wt.\% Zn ingot, at $90 \mathrm{~mm}$ from the chill. positioned around the tips. Two twinned arms are growing parallel to the twin plane at $60^{\circ}$ on each side of the trunk, while two other arms, also at $60^{\circ}$ from the trunk, are growing on each side of the twin plane, but at $\pm 70.5^{\circ}$ from this plane (indicated in Fig. 1a).

The stability of twinned dendrites during a Bridgman experiment was assessed independently of their interactions with columnar dendrites, since a small cylindrical seed was machined out of a fully twinned region of a DS ingot. A typical macrostructure of a twinned Al-23 wt.\% $\mathrm{Zn}$ specimen remelted, then resolidified at $v_{\mathrm{p}}=6.6 \times$ $10^{-5} \mathrm{~m} \mathrm{~s}^{-1}$ and finally quenched in a water bath is shown in Figure 2. Different zones of the sample have been identified in the figure: (i) on the right, the zone of the seed specimen where partial remelting has occurred; (ii) on the left, the quenched liquid after regrowth at $v_{\mathrm{p}}$; and (iii) in the middle, the quenched microstructure that has formed during regrowth. Before focusing on this last part of the specimen, let us briefly discuss the remelted part of the seed. As microsegregation occurs during DS solidification, soluterich regions melt first. Note that the solid-liquid interface during remelting is not necessarily at equilibrium due to slow diffusion in the solid $[12,13]$. Furthermore, the solidstate transformations that can occur below the monotectoid temperature of $\mathrm{Al}-\mathrm{Zn}$ might complicate this picture. Simultaneously with remelting, back-diffusion in the solid phase occurs with a diffusion coefficient $D_{s}$, which is a function of the local temperature and composition. The associated Fourier number, $4 D_{s}\left(t_{\mathrm{p}}-t_{0}\right) / \lambda_{2}^{2}$, where $\lambda_{2}$ is the secondary dendrite arm spacing, is on the order of unity. This contributes to the homogenization of the composition in the solid, and therefore helps to slow down the remelting operation. In regions where isolated droplets have formed, a process called thermal gradient zone melting [14] occurs. In a thermal gradient, such droplets tend to migrate in the direction of $G$, but at fairly low speed. Under the present conditions, the migration velocity was estimated to be $1 \times 10^{-7} \mathrm{~m} \mathrm{~s}^{-1}$, which means that within the time $\left(t_{\mathrm{p}}-t_{0}\right)$, liquid droplets have moved by no more than $100 \mu \mathrm{m}$. This phenomenon can therefore be ignored in the present case. In regions where the liquid is continuous, the main evolution mechanism is coarsening and diffusion. Because the temperature gradient induces a solute gradient in the liquid, the typical diffusion distance (without convection) associated with time $\left(t_{\mathrm{p}}-t_{0}\right)$, i.e. $\sqrt{D_{\ell}\left(t_{\mathrm{p}}-t_{0}\right)}$, is typically 1-2 mm, which is comparable to the scale shown in Figure 2. As solute diffuses in the direction of the solute gradient $G$, indicated with an arrow, this has a tendency to remelt the hot solid and solidify the cold region. This also induces a positive segregation in the hot region and a negative segregation in the cold one. All these complex and interconnected phenomena lead to the various contrasts seen in Figure 2, the dark/light regions corresponding to zinclean/rich regions in this scanning electron microscopybackscattered electron (SEM-BSE) micrograph (i.e. the intensity is proportional to the Z-number of the element). A composition profile along the thermal gradient, measured by energy-dispersive X-ray analysis (EDX) on lines perpendicular to $G$ is shown at the top of the figure. As can be seen, the quenched liquid has a "nominal" composition of $23 \mathrm{wt} . \%$, whereas the regrowth zone and the partially remelted zone are slightly leaner and richer in zinc, respectively. 

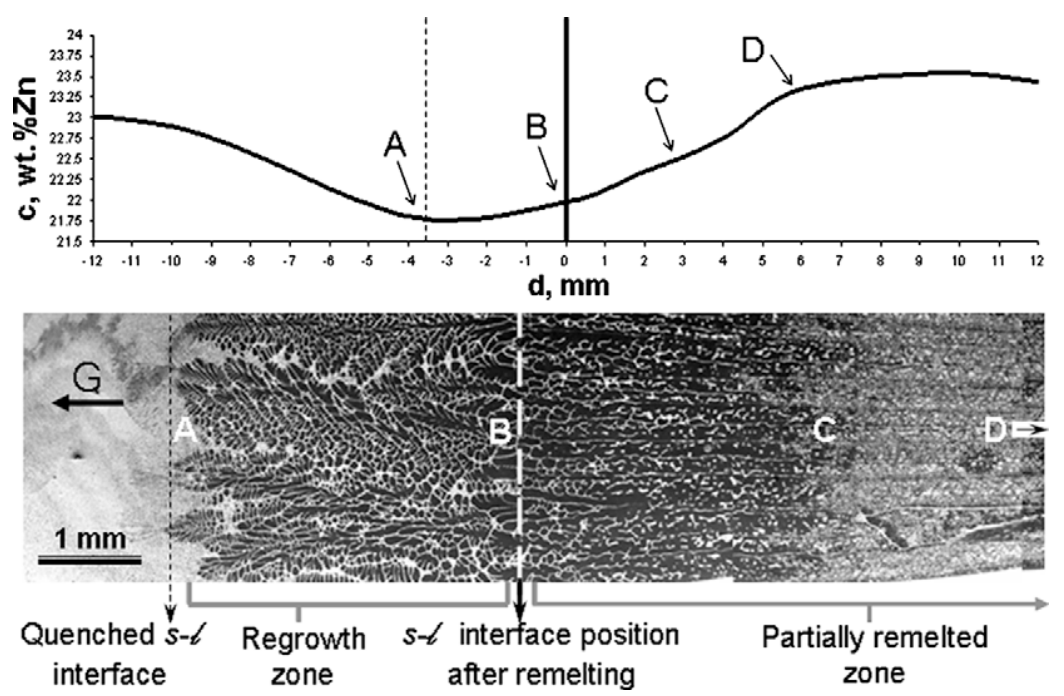

Figure 2. Macrostructure of an Al-23 wt. $\% \mathrm{Zn}$ twinned specimen partially remelted and then resolidified at $v_{\mathrm{p}}=6.6 \times 10^{-5} \mathrm{~m} \mathrm{~s}^{-1}$. A composition profile measured by EDX is shown on the top.

Once regrowth from the partially remelted seed starts, the actual distance of growth does not correspond to $v_{\mathrm{p}}\left(t_{\mathrm{q}}-t_{\mathrm{p}}\right)$. In fact, if $\mathrm{C}_{l 0}$ is the composition of the fully liquid region at the onset of pulling, the solute layer that has to build up during regrowth makes the solid-liquid front first recede before it destabilizes and gives dendrites growing under a steady state at a velocity $v_{\mathrm{p}}$ just below the liquidus $\mathrm{T}_{l i q}\left(\mathrm{C}_{l 0}\right)$.

Figure 3 a shows an enlarged view of the transition zone between the partially remelted seed and the resolidified region. Again, the Al-dendrites appear as dark, with the zincrich interdendritic regions appearing light. Two coherent twin planes have been outlined with a straight white line, whereas the transition between the remelted and resolidified regions is marked with a dashed black line. The location of the remelting front was confirmed by partially remelting another specimen under the same conditions and then directly quenching it without any growth sequence. Despite the fact that the specimen temperature in this region is in between the liquidus and solidus of the alloy, the twin planes have remained present in the partially remelted region. The square in Figure 3a is a region where EBSD measurements were performed. The false grey-level reconstructed EBSD micrograph in Figure $3 \mathrm{~b}$ was obtained using the angle between the thermal gradient direction (vertical in this figure) and the closest $\left\langle\begin{array}{llll}1 & 1 & 0\end{array}\right\rangle$ direction. The $\left\langle\begin{array}{lll}1 & 1 & 0\end{array}\right\rangle$ pole figure in Figure $3 \mathrm{c}$ has the same axes as the micrographs, i.e. the normal to the section is at the center of the projection and $G$ is vertical. The (llll 111$)$ twin plane is indicated by an arc of a circle, while the three $\left\langle\begin{array}{llll}1 & 1 & 0\end{array}\right\rangle$ directions shared by the twinned and untwinned regions are represented by half-filled squares. The three other $\left\langle\begin{array}{lll}1 & 1 & 0\end{array}\right)$ directions of these two zones of this twinned grain are represented as filled (for dark grey regions) and open squares (for light grey regions). As can be seen from Figure 3b, solidification at $v_{\mathrm{p}}=3.3 \times 10^{-4} \mathrm{~m} \mathrm{~s}^{-1}$ induces epitaxial growth from the partially remelted seed, i.e. twinned and untwinned regions with the same crystallographic orientation as the parent zones of the seed can be observed in the resolidified zone. However, the dark regions have clearly grown at the expense of the light ones and, moreover, the transitions between them no longer correspond to coherent twin planes. This indicates that, under the present conditions, regular dendrites have a growth advantage over twinned morphologies, even with the pre-existence of twinned planes.

Indeed, in Figure 3a, a regular dendritic morphology is observed within each resolidified region issued from the twinned and untwinned parts of the seed. This is particularly evident in Figure 4, which shows an enlarged view of the regrowth zone of a similar specimen resolidified at $v_{\mathrm{p}}=6.6 \times 10^{-5} \mathrm{~m} \mathrm{~s}^{-1}$. The pole figures on the left correspond to the $\left\langle\begin{array}{llll}1 & 0 & 0\end{array}\right\rangle$ (circles) and $\left\langle\begin{array}{llll}1 & 1 & 0\end{array}\right\rangle$ (squares) directions of regions $\mathrm{A}$ and $\mathrm{B}$, issued from the untwinned (filled symbols) and twinned parts (filled symbols) of the seed, respectively. While the twin relationship is still evident between these two zones, it is also clear that the separation between them no longer corresponds to a coherent twin plane (white vertical curved line going in between the white and black crosses). Looking more closely at regions $\mathrm{A}$ and $\mathrm{B}$, the growth direction of regular dendrites can be identified: the direction of the primary trunk and secondary arms has been identified by black, respectively, white, lines in the micrograph. Their orientation is shown in the corresponding pole figure with a heavy square and circle(s). In both cases, it appears that the trunk of these regular dendrites has grown along the $\left\langle\begin{array}{llll}1 & 1 & 0\end{array}\right\rangle$ direction closest to the vertical thermal gradient, whereas secondary arms have grown along $\left\langle\begin{array}{lll}1 & 0 & 0\end{array}\right\rangle$ directions, which are seen at either $45^{\circ}$ (zone A) or $90^{\circ}$ (zone B) from the trunks.

Based on these findings, the following conclusions can be drawn:

- The formation of twinned dendrites in aluminum alloys is not only related to the ability to nucleate a stacking fault. Indeed, even if twinned planes are already present in a partially remelted seed, regrowth does not induce the formation of such morphologies. Instead, independent regular dendrites grow epitaxially from the twinned and untwinned parts of the seed. They compete in the temperature gradient as regular dendrites and after solidification form a particular grain boundary with an incoherent twin relationship. 

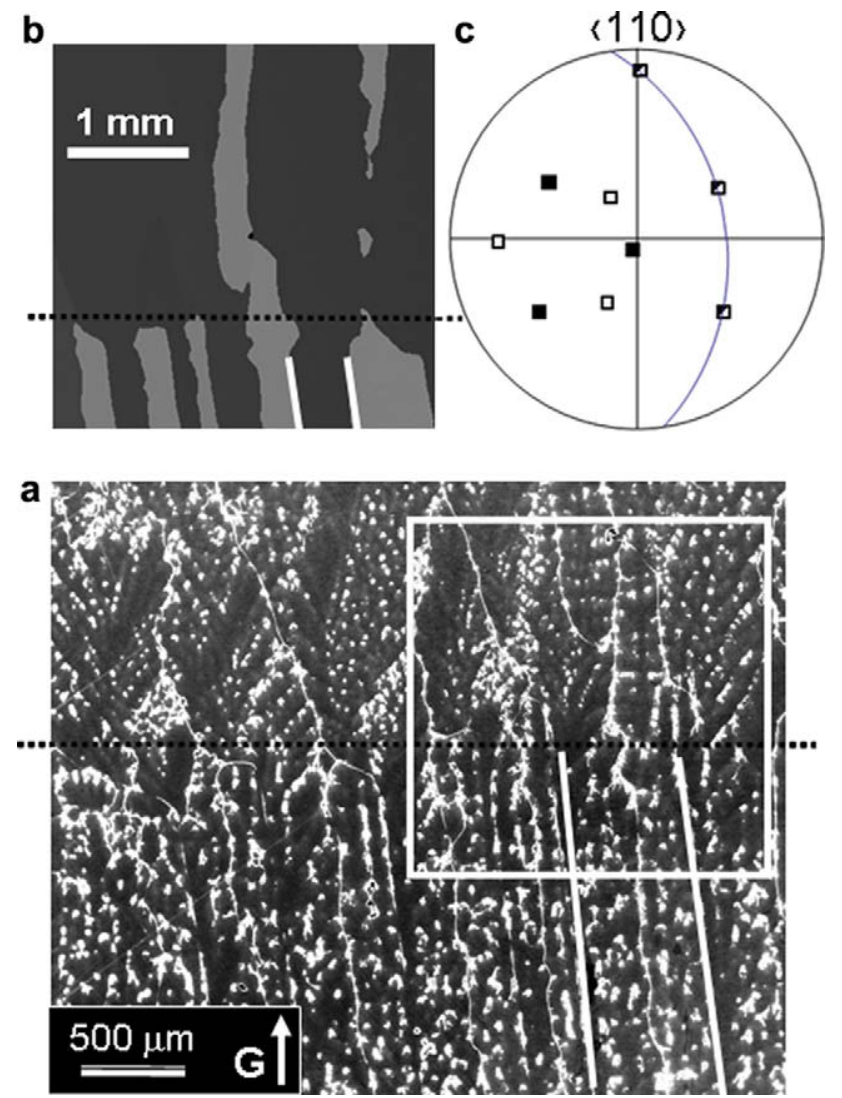

Figure 3. (a) SEM-BSE micrograph of the longitudinal section of a twinned $\mathrm{Al}-23 \mathrm{wt} \% \mathrm{Zn}$ specimen partially remelted and then resolidified at $v_{\mathrm{p}}=3.3 \times 10^{-4} \mathrm{~m} \mathrm{~s}^{-1}$. (b) False grey-level reconstructed EBSD micrograph of the square region shown in (a). (c) $\langle 110\rangle$ pole figure of the twinned grain with the normal to the section placed at the center of the projection and $G$ vertical. The coherent $\left\{\begin{array}{llll}1 & 1 & 1\end{array}\right\}$ twin plane is indicated with an arc of a circle, the three $\left\langle\begin{array}{llll}1 & 1 & 0\end{array}\right\rangle$ directions shared between the twinned and untwinned regions being shown by half-filled squares. The three other $\left\langle\begin{array}{llll}1 & 1 & 0\end{array}\right\rangle$ directions of the dark/light grey regions in (b) are shown by filled/open squares.

- The propagation of a twinned morphology is not favored in the Bridgman specimen for two reasons: the thermal gradient and the convection in the melt are greatly reduced compared to the parent DS specimen. It is unlikely, however, that the effect of a reduced $G$ alone inhibits the propagation of twinned dendrites since, in the DS sample, twin growth can occur up to nearly the top of the ingot, where the thermal gradient is comparable to that of the Bridgman furnace.

- In the regrowth region, the direction of the primary trunk of regular dendrites is $\langle 110\rangle$, even though it was shown that $\langle 100\rangle$ regular dendrites are expected for such compositions $[11,15]$. However, it should be kept in mind that $\left\langle\begin{array}{llll}1 & 0 & 0\end{array}\right\rangle$ growth directions were observed in Al-Zn below 25 wt.\% when free competition was allowed to take place among many grains of random orientation. In the present case, regrowth was forced to take place from a twinned seed, in which a $\left\langle\begin{array}{llll}1 & 1 & 0\rangle & \text { direction was nearly aligned with the thermal }\end{array}\right.$ gradient. Furthermore, the present composition is also close to that where regular dendrites start to deviate from $\left\langle\begin{array}{lll}1 & 0 & 0\end{array}\right.$. It should be recalled that, just above $25 \mathrm{wt} . \%$, textured seaweeds were observed [11,15],
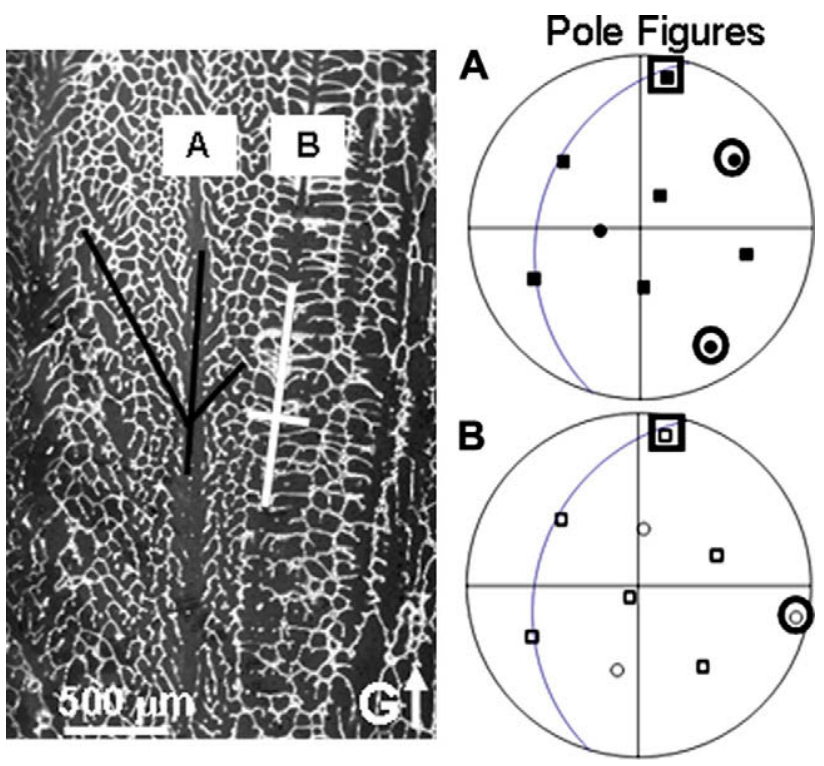

Figure 4. SEM-BSE micrograph of a longitudinal section of a twinned Al-23 wt. $\% \mathrm{Zn}$ specimen partially remelted and then resolidified at $v_{\mathrm{p}}=6.6 \times 10^{-5} \mathrm{~m} \mathrm{~s}^{-1}$, showing the regrowth region (left). On the right, pole figures of zones $\mathrm{A}$ and $\mathrm{B}$ showing the $\left\langle\begin{array}{lll}1 & 0 & 0\end{array}\right\rangle$ (circles) and $\left\langle\begin{array}{lll}1 & 1 & 0\end{array}\right\rangle$ (squares) directions of the regrown dendrites, with the original $\left\{\begin{array}{lll}1 & 1 & 1\end{array}\right\}$ twin plane trace of the seed ( $\operatorname{arc}$ of circle). The heavy squares/circles correspond to the directions of dendrites trunks/secondary arms.

which indicate that the solid-liquid interfacial energy anisotropy is weak. Under such conditions, the thermal gradient can strongly influence the direction of growth of the dendrite trunks. Remarkably, the direction of growth of the secondary arms is $\langle 100\rangle$, in agreement with observations made previously $[11,15]$.

The authors thank the staff of the Interdisciplinary Center for Electron Microscopy at the Ecole Polytechnique Fédéral de Lausanne (EPFL), and are grateful for the valuable technical assistance of J.-D. Wagnière.

[1] J. Herenguel, Rev. Metall. 45 (1948) 339.

[2] S. Henry, P. Jarry, P.-H. Jouneau, M. Rappaz, Metall. Mater. Trans. 28 (1997) 207.

[3] S. Henry, P. Jarry, M. Rappaz, Metall. Mater. Trans. A 29 (1998) 2807.

[4] S. Henry, T. Minghetti, M. Rappaz, Acta Mater. 46 (1998) 2495.

[5] M.A. Salgado-Ordorica, M. Rappaz, Acta Mater. 56 (2008) 5708.

[6] K.I. Dragnevski, R.F. Cochrane, M. Mullis, Metall. Mater. Trans. A 35 (2004) 3211.

[7] S. Henry, G.U. Gruen, M. Rappaz, Meter. Mater. Trans. A 35 (2004) 495.

[8] J.A. Eady, L.M. Hogan, J. Cryst. Growth 23 (1974) 129.

[9] H.J. Wood, J.D. Hunt, P.V. Evans, Acta Mater. 45 (1997) 569.

[10] S. Henry. Ph.D. Thesis, Ecole Polytechnique Fédéral de Lausanne, 1999.

[11] F. Gonzales, M. Rappaz, Metall. Mater. Trans. A 37 (2006) 2797.

[12] T.A. Lograsso, A. Hellawell, J. Cryst. Growth 66 (1984) 531.

[13] M. Rettenmayr, O. Warkentin, M. Rappaz, H.E. Exner, Acta Mater. 49 (2001) 2499.

[14] M.P. Watson, J.D. Hunt, Metall. Trans. A 8 (1977) 1793.

[15] F. Gonzales, M. Rappaz, Metall. Mater. Trans. A 39 (2008) 2148. 